\title{
Prediction of antiepileptic drug treatment outcomes using machine learning
}

\author{
Sinisa Colic, Robert G. Wither, Min Lang, Zhang Liang, James H. \\ Eubanks, and Berj L. Bardakjian,
}

Version Post-print/accepted manuscript

Citation Colic, S., Wither, R. G., Lang, M., Zhang, L., Eubanks, J. H., \& (published version) Bardakjian, B. L. (2016). Prediction of antiepileptic drug treatment outcomes using machine learning. Journal of neural engineering, 14(1), 016002.

Publisher's Statement This is an author-created, un-copyedited version of an article accepted for publication/published in [insert name of journal]. IOP Publishing Ltd is not responsible for any errors or omissions in this version of the manuscript or any version derived from it. The Version of Record is available online at 10.1088/1741-2560/14/1/016002.

How to cite TSpace items

\begin{abstract}
Always cite the published version, so the author(s) will receive recognition through services that track citation counts, e.g. Scopus. If you need to cite the page number of the author manuscript from TSpace because you cannot access the published version, then cite the TSpace version in addition to the published version using the permanent URI (handle) found on the record page.
\end{abstract}

This article was made openly accessible by $U$ of $T$ Faculty. Please tell us how this access benefits you. Your story matters. 


\begin{abstract}
Objective. Antiepileptic drug (AED) treatments produce inconsistent outcomes, often necessitating patients to go through several drug trials until a successful treatment can be found. This study proposes the use of machine learning techniques to predict epilepsy treatment outcomes of commonly used AEDs. Approach. Machine learning algorithms were trained and evaluated using features obtained from intracranial electroencephalogram (iEEG) recordings of the epileptiform discharges observed in Mecp2-deficient mouse model of the Rett Syndrome. Previous work have linked the presence of cross-frequency coupling $\left(I_{C F C}\right)$ of the delta $(2-5 \mathrm{~Hz})$ rhythm with the fast ripple $(400-600 \mathrm{~Hz})$ rhythm in epileptiform discharges. Using the $I_{C F C}$ to label post-treatment outcomes we compared Support Vector Machines and Random Forest machine learning classifiers for providing likelihood scores of successful treatment outcomes. Main results. a) There was heterogeneity in AED treatment outcomes, b) machine learning techniques could be used to rank the efficacy of AEDs by estimating likelihood scores for successful treatment outcome, c) I IFC features yielded the most effective a priori identification of appropriate AED treatment, and d) both classifiers performed comparably. Significance. Machine learning approaches yielded predictions of successful drug treatment outcomes which in turn could reduce the burdens of drug trials and lead to substantial improvements in patient quality of life.
\end{abstract}

\section{INTRODUCTION}

$\mathrm{E}_{\mathrm{a}}^{\mathrm{p}}$ PILEPSY is a major neurological disorder that afflicts approximately $1.8 \%$ of the population [1]. Its cause can be attributed to many factors ranging from head trauma to genetic abnormalities. There are more than two dozen commonly used antiepileptic drugs (AEDs), and many more experimental drugs, available to treat the disorder. Determining the most effective treatment for a specific individual often involves a trial-and-error procedure. Furthermore, $20-40 \%$ of epileptic patients develop medically refractory (drug resistant) forms of epilepsy [2, 3], though they only become aware of this after having already participated in numerous AED trials.

Antiepileptic drugs can also make the seizures worse and more frequent, and are associated with numerous side-effects that can affect cognition and patients' abilities to perform [4-

\footnotetext{
Manuscript sent ___ Asterisk indicates corresponding author.

*S. Colic is with the Department of Electrical and Computer Engineering, University of Toronto, Toronto, ON Canada M5S-3G4 (email: sinisa.colic@utoronto.ca)

M. Lang is with the Division of Genetics and Development, University of Toronto Epilepsy Research Program, University of Toronto, Toronto, ON, Canada M5S-1A8

R. G. Wither is with the Department of Physiology, University of Toronto, Toronto, ON, Canada M5S-1A8
}

6]. Furthermore, patients can build up a tolerance to certain drugs over time [7], at which point the trial-and-error search for a treatment resumes.

Unsuccessful drug trials and delayed treatments highly impact patients' quality of life and are expensive for both patients and the health care system. Determining a priori the most effective treatment would go a long way in improving the lives of patients and reducing the financial burden. The first step for developing a successful treatment plan is accurately diagnosing or characterizing the disorder.

In recent years, some progress was made on diagnosing and tracking epileptogenesis for those with medically refractory epilepsy. These studies have provided substantial knowledge on seizure characterization, particularly studies focusing on high frequency oscillations (HFOs). Ripple $(80-200 \mathrm{~Hz})$ and fast ripple (>200 Hz) HFOs were found to coincide with seizure episodes [8-10], indicate seizure onset zones [9-11] and vary in intensity corresponding to AED dosages [12]. However, the presence of HFOs alone is not indicative of pathology. Recent work by Guirgis et al. highlights that HFO presence is mainly linked to pathology when other rhythms are present, specifically the delta $(<4 \mathrm{~Hz})$ rhythm Their findings reveal that delta modulation of HFOs emerges at seizure onset and termination, implying that the delta-HFO modulation may be involved in transition mechanisms leading to epileptogenesis [8]. There is an opportunity to further explore the effect of these biomarkers on predicting epilepsy treatment outcome through machine learning methods.

Pharmacological treatment prediction through the use of machine learning methods has previously been applied on major depressive disorders and schizophrenia [13, 14]. The results show that scalp EEG features examining combinations of power, coherence and mutual information measures are effective at predicting favorable treatment response to antidepressant drug therapy. While machine learning techniques have been applied in epilepsy studies to classify seizure events [15], predict the seizure onset [16], and most recently, identify seizure onset zone [17], machine learning methods that predict treatment outcome have not, to the best

L. Zhang is with the Division of Fundamental Neurology, Department of Medicine, and University of Toronto Epilepsy Research Program, University of Toronto, Toronto, ON, Canada M5S-1A8

J. H. Eubanks is with the Division of Genetics and Development, Department of Surgery, and University of Toronto Epilepsy Research Program, University of Toronto, Toronto, ON, Canada M5S-1A8

B. L. Bardakjian is with the Department of Electrical and Computer Engineering, and Institute of Biomaterials and Biomedical Engineering, University of Toronto, Toronto, ON, Canada M5S-3G9 

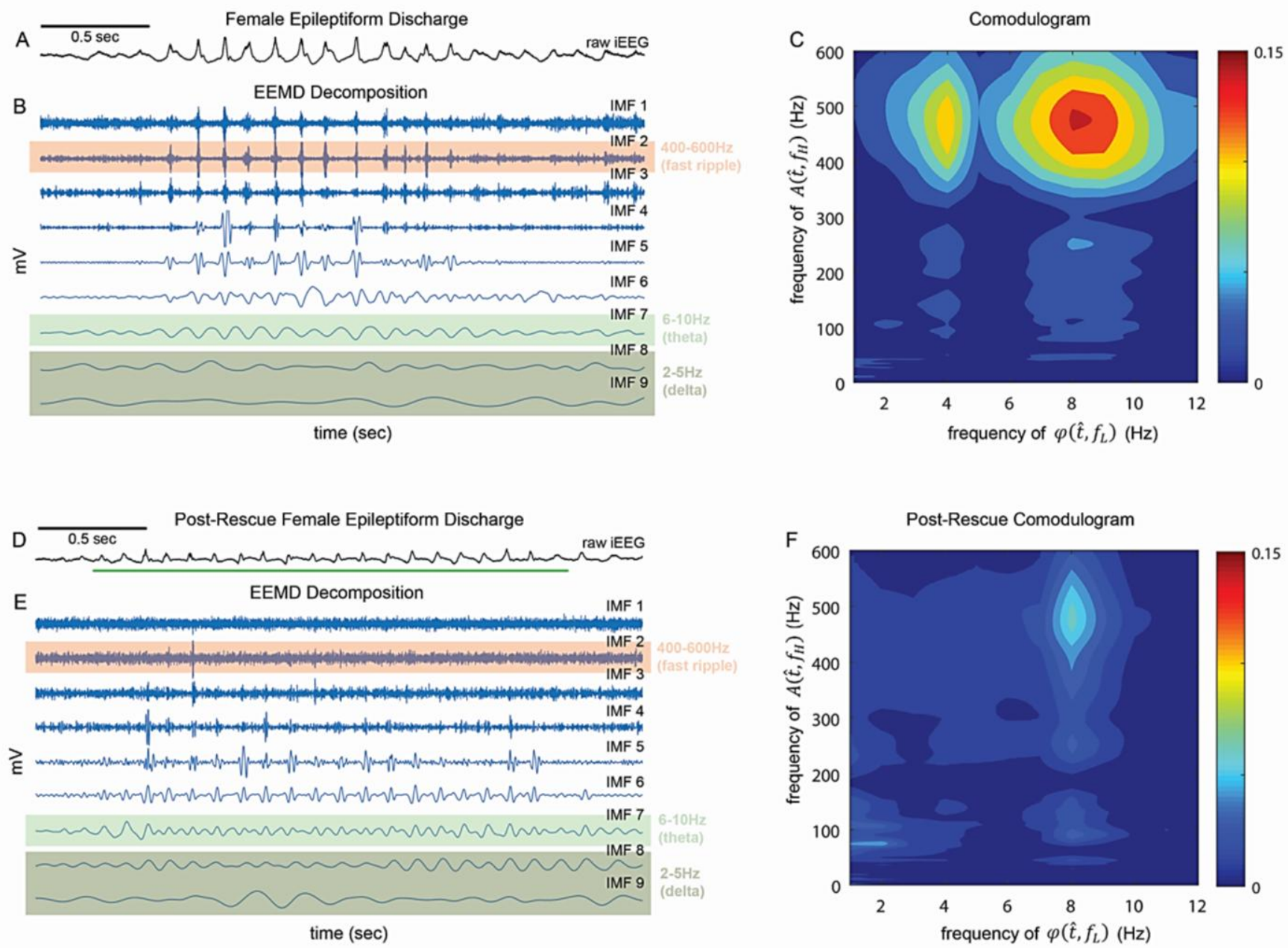

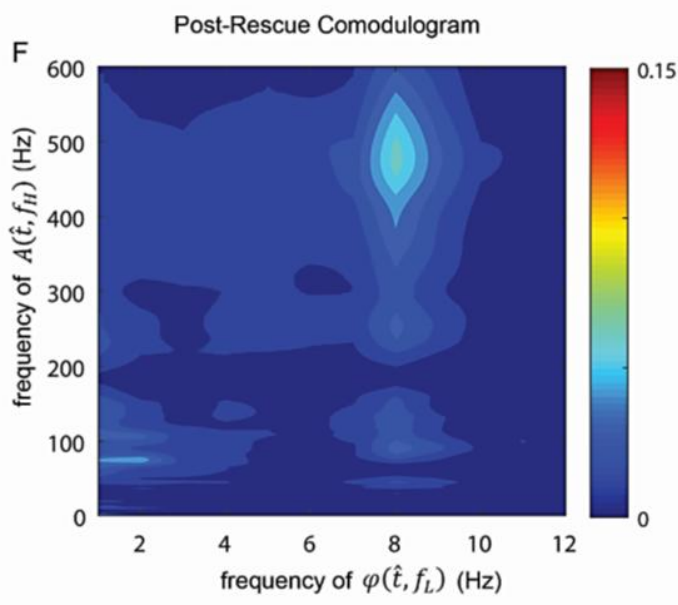

Fig. 1. Characterization of epileptiform discharges in Mecp2-deficient mice pre- and post- mecp2 gene reactivation which were also used for feature extraction and labeling needed for machine learning approaches. A) Time-series recording of typical pre-rescue discharge. B) Frequency decomposition using the EEMD algorithm is able to extract the theta, delta and fast ripple rhythms from the raw iEEG trace. C) Comodulograms showing cross-frequency phase-amplitude coupling prior to mecp 2 gene reactivation shows presence of theta $(6-10 \mathrm{~Hz})$ and delta $(2-5 \mathrm{~Hz})$ modulation with the fast ripple $\mathrm{HFO}(400-600 \mathrm{~Hz})$. D) Timeseries recording of typical post-rescue discharge. E) Frequency decomposition using the EEMD algorithm shows reduction in fast ripple modulation from the raw iEEG trace post gene reactivation. F) Comodulograms showing cross-frequency phase-amplitude coupling post mecp 2 gene reactivation shows reduction in theta $(6-10 \mathrm{~Hz})$ and abolishment of delta $(2-5 \mathrm{~Hz})$ modulation with the fast ripple HFO $(400-600 \mathrm{~Hz})$
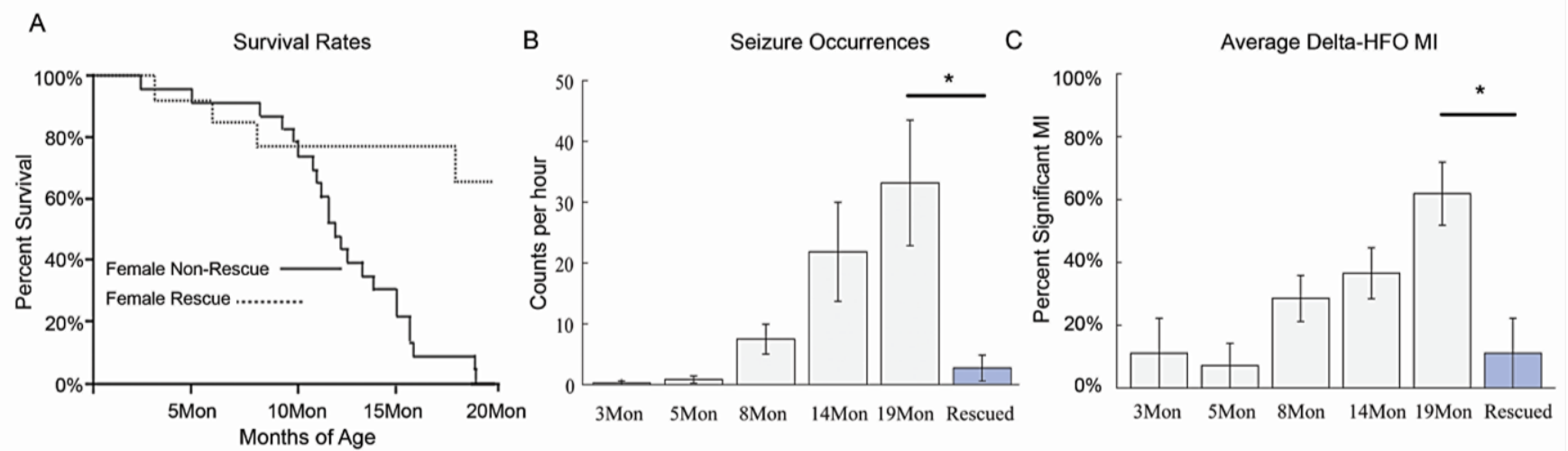

Fig. 2. Comparison of life spans, durations and phase-amplitude cross-frequency coupling pre and post mecp2 gene reactivation. A) Lifespan of Mecp2deficient mice is improved post mecp 2 reactivation. B) Occurrences of discharges show a steady increase with age of animals and is significantly reduced post mecp2 gene reactivation. C) Percentage of discharges exhibiting delta-HFO cross-frequency coupling is significantly reduced post mecp2 gene reactivation. 
of our knowledge, been applied in epilepsy research until now.

In this study we focus on a genetic model of epilepsy, found in Rett Syndrome, which is typically unresponsive to common antiepileptic medication. We show that machine learning (ML) methods that use pre-treatment intracellular electroencephalogram (iEEG) recordings can predict the response to common AEDs.

Rett syndrome is one of the leading genetic causes of severe mental retardation in females and it has been linked to mutations in the gene encoding methyl-CpG-binding protein 2 (MeCP2) [18]. Rett syndrome is characterized by a 6-18 month period of normal development, followed by rapid regression of acquired skills leading to a long list of symptoms which includes epileptic seizures [19]. Epileptic seizures are found to occur in 60-80\% of Rett syndrome patients and the seizures are typically poorly controlled by classic anti-convulsive drugs [19, 20].

Although MeCP2 protein is expressed throughout the body, it is relatively more abundant in the brain [21]. The accepted view of $\mathrm{MeCP} 2$ function is that it acts to repress genetic transcription through binding of methylated $\mathrm{CpG}$ sites and subsequent recruitment of co-repressors. It is also thought that $\mathrm{MeCP} 2$ may be necessary for maintenance of mature neurons rather than their development [22].

Rodent models lacking mecp 2 function have been shown to reproduce many of the behavioral and neurological deficits observed clinically in Rett syndrome patients [23]. Recordings of iEEGs from Mecp2-deficient mice have revealed spontaneous low frequency oscillations (LFO) in the form of epileptiform discharges in the 6-10 $\mathrm{Hz}$ frequency range (Figure 1a,d) [24-26].

Previous work on female Mecp2-deficient mice, building on the findings of the delta-HFO co-modulation showed that the reintroduction of the mecp 2 gene in gene knockout mice results not only in behavioral and physical improvements, but also in the reduction of delta-HFO coupling for discharges greater than 2 seconds in duration (Figure 2) [27].

This study proposes the use of iEEG-based features to track delta-HFO coupling in Mecp2-deficient mice undertaking antiepileptic drug therapy to determine treatment efficacy. Two commonly used machine learning techniques, Support Vector Machines (SVMs) and Random Forests (RF) are trained and evaluated on time-based, normalized power and cross-frequency coupling features to predict the likelihood of treatment outcome for commonly used AEDs.

\section{METHODS}

Figure 3 provides an outline of the experimental setup, machine learning methodology including feature extraction, labeling, training and testing used in this study.

\subsection{Experimental Setup and Data Acquisition}

All animal experimental procedures were approved by local ethics committees in accordance to guidelines of the Canadian Council on Animal Care.
Experimental genotypes were produced by crossing female Mecp2 $^{+/-}$mice $\left(M e c p 2^{\text {tm1.1Bird }}\right.$, Jackson Laboratory, Bar Harbor, ME) with male wild-type mice described previously $[28,29]$. In total there are $(n=6)$ female Mecp $2^{-/+}$subjects. In the case of the gene reactivated model the mice were generated by crossing female Mecp $2^{+/-}$mice with male Rosa26-Esr/Cre transgenic mice (Gt(ROSA) ${ }^{26 \operatorname{Sortml}(\mathrm{cre} / \mathrm{ESR} 1) \mathrm{Tyj} / \mathrm{J}}$ , Jackson Laboratories) [29, 30]. In total there are $(n=4)$ female mecp 2 reactivated mice (rescued mice). All subjects were maintained on a pure C57BL/6 background and housed in a vivarium that was maintained at $22-23^{\circ} \mathrm{C}$ with a standard 12-hour light cycle commencing at 06:00.

Animals were implanted with stainless steel, polyimideinsulated stainless steel electrodes $(125 \mu \mathrm{m})$ following procedures described previously [25, 31]. Preconfigured microelectrodes were implanted in the somatosensory cortex (Bregma, - $0.8 \mathrm{~mm}$; lateral, $1.8 \mathrm{~mm}$; depth, $1.5 \mathrm{~mm}$ ) with a reference implanted superficially in the frontal cortex (Bregma $+2.8 \mathrm{~mm}$; lateral, $1.8 \mathrm{~mm}$; depth, $0.5 \mathrm{~mm}$ ). iEEG signals were amplified 1000x, bandpass-filtered (0.01 - 1000 $\mathrm{Hz}$ ) and digitized (Digidata 1300, Axon Instruments, Weatherford, TX, USA). Data were sampled at $60 \mathrm{kHz}$ and stored using Clampfit 10.2 software (Axon Instruments). Recordings sessions lasted from $30 \mathrm{~min}$ to $1 \mathrm{~h}$ to observe all of the behavior states.

Baseline was taken to indicate Mecp2-deficient female mice in the 19-23 months age range. All pharmacological treatments were applied for a period of one day followed by a day of washout. The drugs and dosages used in this study were: Midazolam at $0.5 \mathrm{mg} / \mathrm{kg}$, Ganaxolone at $5 \mathrm{mg} / \mathrm{kg}$, THIP at $5 \mathrm{mg} / \mathrm{kg}$, and Phenytoin at $30 \mathrm{mg} / \mathrm{kg}$. All drugs were dissolved in double distilled $\mathrm{H}_{2} \mathrm{O}$ and administered intraperitoneally to the animals.

Recordings of iEEG were obtained from Mecp $2^{+/-}$mice exhibiting spontaneous and recurrent epileptiform discharges. These recordings came from pre- and post- mecp 2 gene reactivation, and pre- and post- anti-epileptic drug (AED) treatment regiments (Figure 3a). Epileptiform events were identified in the recordings using an automated detection technique based on predefined selection criterion as described previously [24, 32]. Data preprocessing consisted of downsampling to $4 \mathrm{k} \mathrm{Hz}$ followed by removal of $60 \mathrm{~Hz}$ line noise using a high order FIR notch filter with $+/-0.5 \mathrm{~Hz}$ cutoffs. Segments with large amplitude muscle artifacts were excluded from analysis.

\subsection{Features for Machine Learning}

In this study we investigate power-based features from the normalized complex wavelet transform (CWT), crossfrequency coupling based comodulogram, and time-based features from extracted rhythms using ensemble empirical mode decomposition (EEMDs) (Figure 3b). Two machine learning algorithms: SVMs and RFs were evaluated on their ability to predict treatment out come from iEEG-based features (Figure 3c). 
A

B

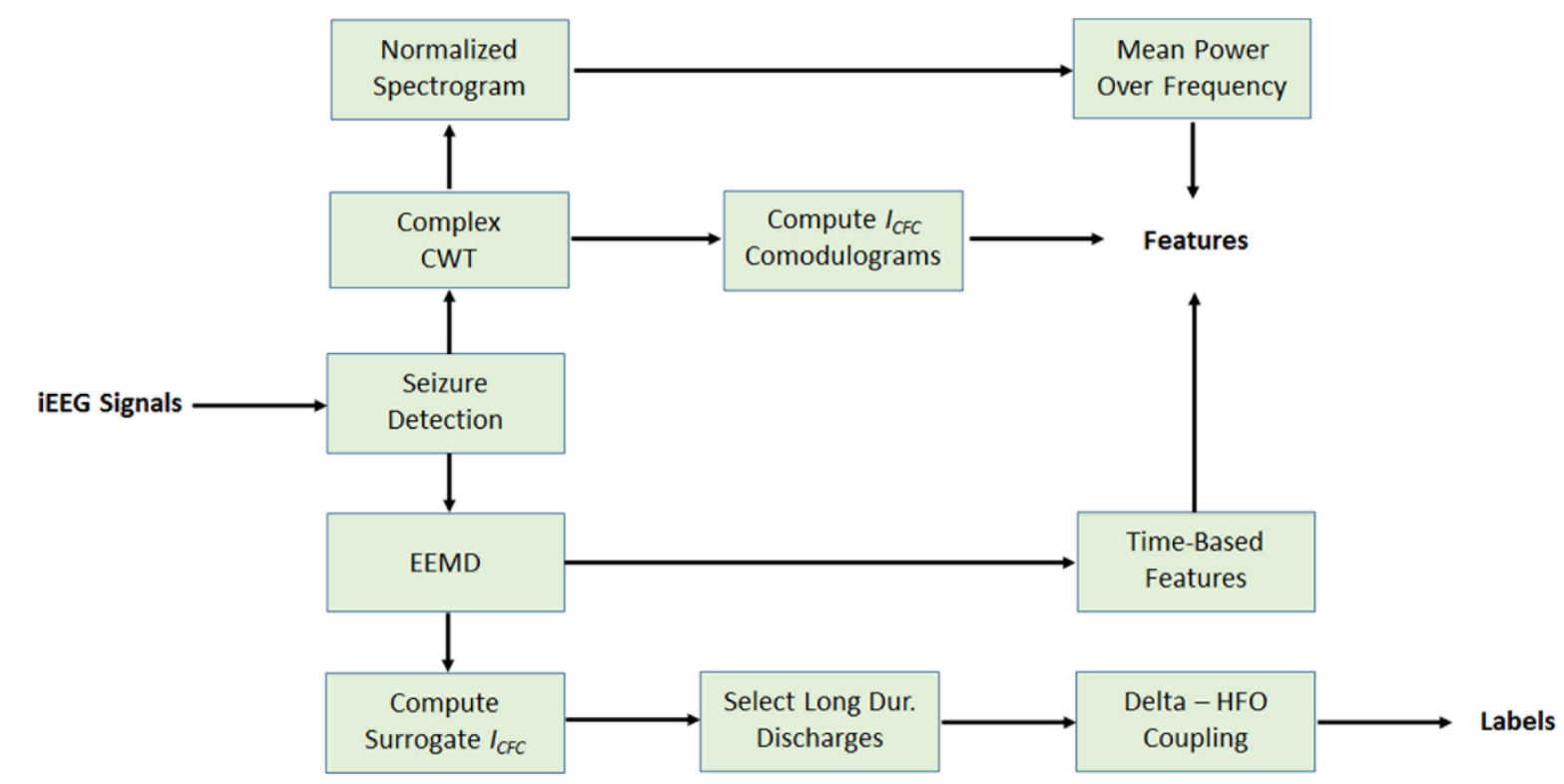

C

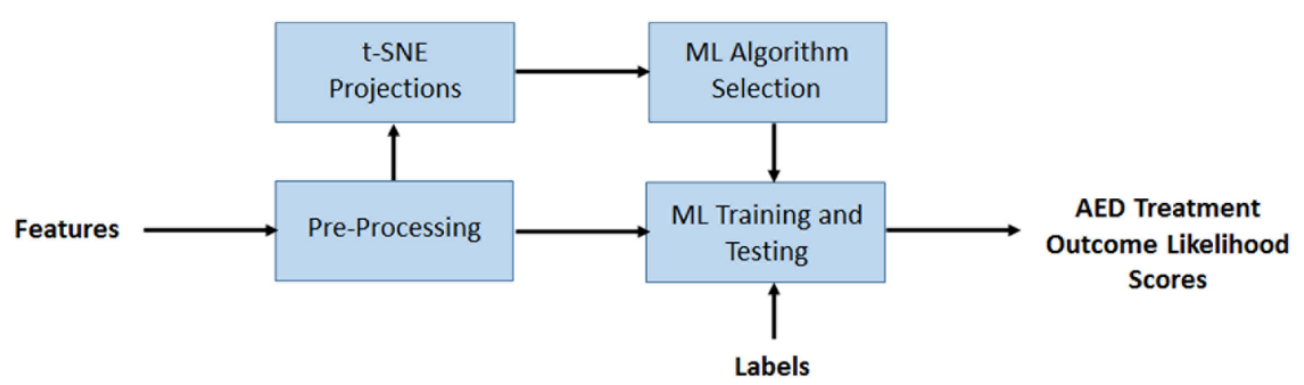

Fig. 3. Overview of experimental design, signal processing and machine learning methods and their interconnection. A) Experimental setup consisted of pre and post gene reactivation along with pharamacological testing. B) Signal processing overview highlights the rhythm extraction using complex CWT and extraction of specific rhythms of interest using EEMD for purposes of generating labels. C) Machine learning overview highlighting the selection of machine learning algorithms using t-SNE projections. 


\subsubsection{Comodulogram}

Time-frequency power distributions were obtained by applying a continuous wavelet transform (CWT) on iEEG time-series recordings $x(\hat{t})$, where $\hat{t}$ is a time interval centered on an epileptiform discharge. The CWT measures the correlation between a signal $x(\hat{t})$, and a wavelet basis, $\psi$, for different scales, $s$, and time shifts $\tau$ [33] and is defined as,

where,

$$
W(s, \tau)=\int_{\hat{t}} x(t) \psi_{s, t}^{*}(t) d t
$$

$$
\psi_{s, t}^{*}(t)=\frac{1}{\sqrt{s}} \psi_{o}\left(\frac{t-\tau}{s}\right)
$$

is the basis function with * denoting the complex conjugate. The basis function used here is the complex Morlet wavelet defined according to the following form,

$$
\psi_{o}(t)=\frac{1}{\sqrt{2 \pi}} \exp \left(i \omega_{c} t-\frac{t^{2}}{2}\right)
$$

The scales were transformed to appropriate frequencies $f$, from the angular frequency $\omega_{c}$, using the relation $\omega_{c}=$ $2 \pi f s=5.1 \mathrm{rad} / \mathrm{s}$. The result of the CWT yielded complex valued coefficient matrix,

$$
W(f, t)=w(f, t)+i \widetilde{w}(f, t)
$$

for which the magnitude was obtained as a measure of power over time and frequency. The rows corresponded to frequencies 1 to $600 \mathrm{~Hz}$ by $1 \mathrm{~Hz}$ step size, and the columns corresponding to the duration of the recording $x(\hat{t})$.

To visualize the high and low frequencies on the same scale, the time-frequency vectors were further $\mathrm{z}$-score normalized according to the following,

$$
W_{\text {norm }}(f, t)=\frac{|W(f, t)|-\left.\mu(f)\right|_{t_{1}} ^{t_{2}}}{\left.\sigma(f)\right|_{t_{1}} ^{t_{2}}}
$$

where variables $\mu$ and $\sigma$ represent the mean and standard deviation from wavelet coefficient magnitudes for each corresponding frequency taken from a two second baseline segment $\hat{b}$ obtained prior to seizure onset (i.e. $\hat{b} \in\left[t_{1}, t_{2}\right]$ ).

Equation 2.2 yields the power-based feature. Normalized CWT power features were obtained for the time windows spanning the duration of the discharges. Power features were obtained by averaging CWT coefficients across time for each frequency band (i.e. $\langle W(f, \hat{t})\rangle)$ from 1 to $600 \mathrm{~Hz}$ resulting in a 600 point feature vector.

Cross-frequency coupling for a time interval $\hat{t}$, is proposed as a composite complex-values signal $S_{C F C}(\hat{t})$ consisting of an amplitude time-series of one higher frequency $A\left(\hat{t}, f_{H}\right)$ with a lower frequency of phase time-series $\varphi\left(\hat{t}, f_{L}\right)$ as shown,

$$
S_{C F C}\left(\hat{t}, f_{H}, f_{L}\right)=A\left(\hat{t}, f_{H}\right) e^{j \varphi\left(\hat{t}, f_{L}\right)}
$$

The time-series of the amplitude envelope $A\left(\hat{t}, f_{H}\right)$ and instantaneous phase $\varphi\left(\hat{k}, f_{L}\right)$ were determined from the respective complex wavelet coefficients,

$$
\begin{gathered}
A\left(\hat{t}, f_{H}\right)=\left|w\left(\hat{t}, f_{H}\right)+j \widetilde{w}\left(\hat{t}, f_{H}\right)\right| \\
\varphi\left(\hat{t}, f_{L}\right)=\arctan \frac{\widetilde{w}\left(\hat{t}, f_{L}\right)}{w\left(\hat{t}, f_{L}\right)}
\end{gathered}
$$

The coupling of the amplitude of a higher frequency signal, $A\left(\hat{t}, f_{H}\right)$ for a phase of a lower frequency signal $\varphi\left(\hat{t}, f_{L}\right)$ was assessed over a range of frequency pairs using the algorithm proposed by Tort et al. [34]. The $\varphi\left(\hat{t}, f_{L}\right)$ signal was segmented into 20 degree intervals resulting in $N=18$ bins. Within each bin the amplitude envelopes were averaged $\left\langle A\left(\hat{t}, f_{H}\right)\right\rangle$. The mean amplitude was normalized by the sum over all mean amplitudes in each phase bin, according to,

$$
p_{j}\left(\hat{t}, f_{H}, f_{L}\right)=\frac{\left\langle A\left(\hat{t}, f_{H}\right)\right\rangle_{j}}{\sum_{k=1}^{N}\left\langle A\left(\hat{t}, f_{H}\right)\right\rangle_{k}}
$$

producing a probability density value $p_{j}$, where $\mathrm{j}$ indicates the phase bin number which is associated with $f_{L}$. Then an entropy measure defined by:

$$
H\left(\hat{t}, f_{H}, f_{L}\right)=-\sum_{j=1}^{N} p_{j}\left(\hat{t}, f_{H}, f_{L}\right) \log \left(p_{j}\left(\hat{t}, f_{H}, f_{L}\right)\right)
$$

was determined and normalized to obtain the index of crossfrequency coupling,

$$
I_{C F C}\left(\hat{t}, f_{H}, f_{L}\right)=\frac{H_{\max }-H\left(\hat{t}, f_{H}, f_{L}\right)}{H_{\max }}
$$

where $H_{\max }$ is the maximum possible entropy value, which for a uniform distribution has a value $H_{\max }=\log \mathrm{N}$.

The $I_{C F C}$ measure described by equation 4.3 when examined over a range of frequencies results in the comodulogram.

Phase-amplitude cross-frequency coupling features were obtained using a comodulogram applied over a 2 second time window centered on the discharge event. The $f_{L}$ spanned 2 $12 \mathrm{~Hz}$, and the $f_{H}$ spanned $80-600 \mathrm{~Hz}$. This resulted in a large feature vector which needed to be compressed vertically to remain similar in size to the other feature sets. The initial feature vector, which had a dimension of 11 by 520, was compressed vertically to 11 by 30 , yielding 330 pixel-based features of the phase-amplitude cross-frequency coupling.

\subsubsection{EEMD Rhythm Extraction}

The Ensemble Empirical Mode Decomposition (EEMD) 
A

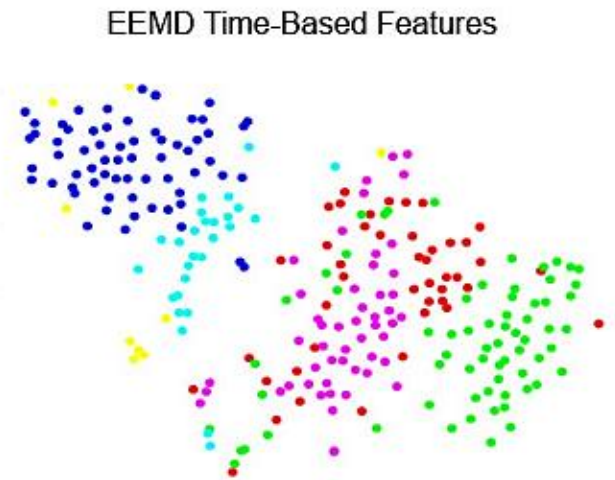

C

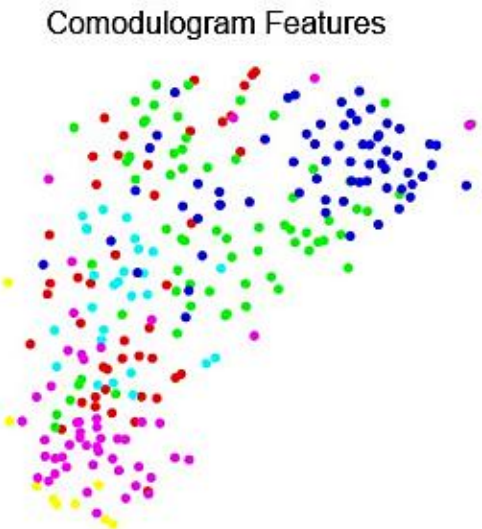

$\mathrm{B}$ Normalized Power Features

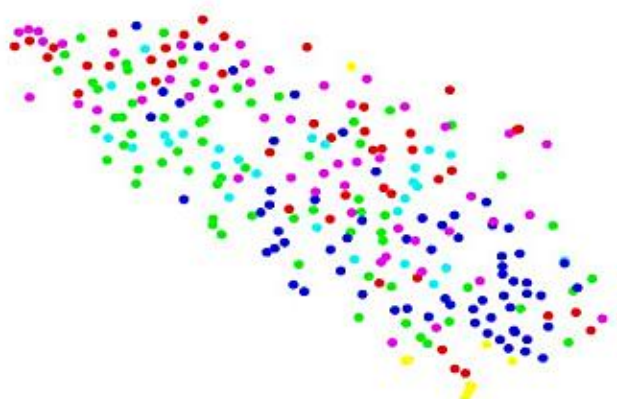

- $\quad$ Mouse 1

Mouse 2

Mouse 3

Mouse 4

Mouse 5

Mouse 6

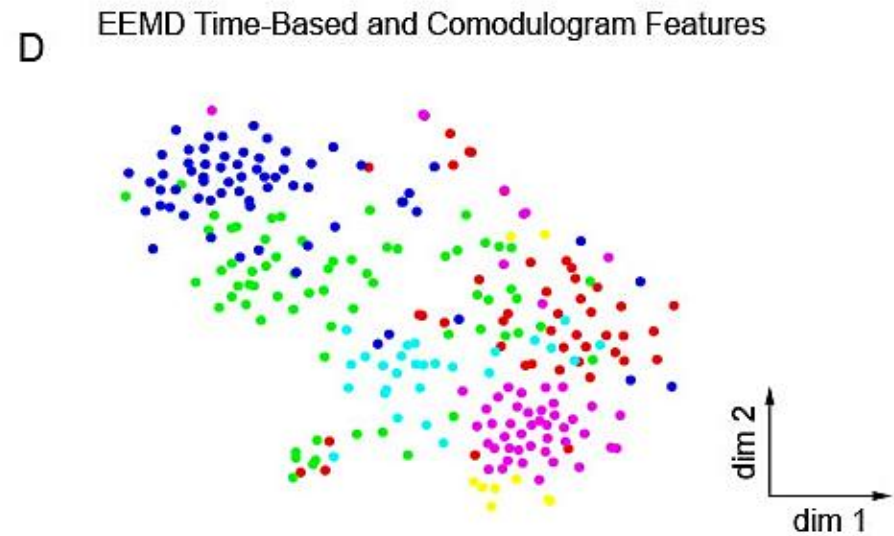

Fig. 4. Low dimensional representation of machine learning features using t-SNE. Each animal subject was identified by a different color. A) EEMD timebased features showed clustering B) Wavelet-based normalized power features were scrambled with little to no clustering reducing possibility for separability of samples C) Comodulogram-based features of phase-amplitude cross-frequency coupling show reasonable clustering D) Concatenating EEMD time-based and comodulogram features showed distinct clustering and strong separability making it the preferred feature set.

was applied to extract the LFO and HFO rhythms [35]. EEMD is applied to decompose signals into rhythms and it does not require a priori knowledge of the frequency ranges of the rhythms, as would have been needed for bandpass filtering. Furthermore, EEMD is better suited for extracting rhythms with time-varying frequencies that span large frequency bands.

The EEMD separates a signal into multiple rhythms referred to as intrinsic mode functions (IMFs). The decomposition is adaptive and dependent on local time characteristics of the data. In our approach the noise variance $\sigma^{2}$ was set to 0.2 , and the number of iterations was set to 100 , resulted in 9 IMFs. The IMFs spanned over a large range of frequencies with IMF1 representing the highest and IMF9 being the lowest frequency rhythm. The theta rhythm LFOs (6-10 $\mathrm{Hz}$ frequency range) were obtained from IMF 8 , the delta LFOs (2-5 Hz frequency range) were obtained by combining IMF8 and IMF9 and the fast ripple HFOs (400 $600 \mathrm{~Hz}$ frequency range) were obtained from the IMF2.

EEMD time-based features were computed for each IMF over time windows $\hat{t}$, spanning the duration of the discharges. The features selected have previously been used in epilepsy studies using machine learning and are formed from a combination of power, RMS power, mean, variance, skewness, kurtosis, line length, zero crossings, non-linear energy, activity, mobility and complexity [36] resulting in an 81 feature vector.

\subsection{Machine Learning Algorithms}

Feature sets were z-score normalized over all samples to reduce the effect of feature scale during training. A two dimensional representation of the features was obtained using t-Distributed Stochastic Neighbor Embedding (t-SNE). The 2-dimensional projections were identified by the subject they came from (Figure 4) and by their response to the AED treatments (Figure 5). The level of separabilty suggests the viability of Support Vector Machines (SVMs) and Random Forest (RF) machine learning classifiers for predicting the treatment outcomes [37].

\subsubsection{Support Vector Machines}

SVMs with Gaussian kernels, implemented in the LibSVM library [38], were trained to predict treatment outcomes for several AEDs. SVMs were chosen for their suitability to small data sets with large feature vectors. SVM work by applying a $\mathrm{n}$-dimensional transformation to construct a hyperplane that maximizes the separation margin between input data classes [39]. 
Training consisted of 5-fold cross-validation to select the best regularization parameter $C$ and the Gaussian parameter $\gamma$. An exponential grid search over the parameters $C\left(2^{-3}, 2^{-4}, \ldots\right.$ , $\left.2^{10}\right)$ and $\gamma\left(2^{-18}, 2^{-17}, \ldots, 2^{-3}\right)$ was then performed. The parameter pair yielding maximum performance on the training set was selected for testing.

\subsubsection{Random Forest}

RF classifier, proposed initially by Breiman [40], was used as one of the predictive models for AED treatment outcome. The RF is made up of individual classification trees. Each tree independently able to make classification decisions. During training a subset of the trees are randomly selected and trained on the dataset. The core of the random forest classifier is the binary decision tree, a data type that stores elements hierarchically in nodes. Each decisions tree is grown on different bootstrapped sample collections (i.e. randomly drawn instances with replacement form the original dataset) on a randomly selected subset of all available predictors. The random selection of predictors increases the generalizability of the individual decisions trees, whereas the collection of multiple decision trees in one forest increases model performance. As a result RFs are well suited to nonlinear, high dimensional feature spaces, and provide a good comparison to SVMs. Furthermore RFs generally work out of the box with minimal parameter searching.

Random Forest (RF) implementation followed similarly to the SVM implementation with less parameter searching. A search over the number of trees was performed from 50 to 400. As it was observed that after 100 trees the performance did not improve, the number of trees was fixed at 100 for the remainder of the testing.

\subsection{Training}

Typical machine learning approaches would warrant some form of feature selection, such as the minimal-redundancymaximal relevance criterion (mRMR)[41]. In this study we maintained feature independence to investigate the contributions of each feature type.

In total there were 263 unique discharges distributed across six subjects along with four feature vectors for each sample. The feature vectors were combined with samples to yield three unique feature matrices (samples $\mathrm{x}$ features) representing the EEMD time-based features, $(263 \times 81)$, normalized CWT power feature, $(263 \times 600)$, and comodulogram features, $(263 \times 330)$. An additional feature set was obtained by joining the EEMD time-based features with the comodulogram features to yield a fourth feature matrix (263x411). This was done to see if the combination of the comodulogram features with the EEMD time-based features would yield any improvements in prediction accuracy.

To maintain independence of training and testing sets, the training sets for each AED were confined to two subjects, one a responder and the other a non-responder. Only half of the available samples were selected for training and equal number of samples from the two classes were used for testing.

\begin{tabular}{|c|cccccc|c|}
\cline { 2 - 8 } \multicolumn{1}{c|}{} & \multicolumn{8}{c|}{ Percentage of Discharges with Delta - HFO Coupling } & Avg. \\
\hline BASELINE & 50 & 67 & 40 & 60 & 100 & 44 & 60 \\
\hline MID & 0 & 54 & 0 & 100 & 100 & 0 & 42 \\
GAN & 33 & 69 & 100 & 38 & 0 & 35 & 46 \\
THP & 54 & 65 & 0 & 57 & 0 & 25 & 33 \\
PHE & 100 & 0 & 100 & 100 & 100 & 25 & 71 \\
\hline
\end{tabular}

Table 1. Examination of delta-HFO phase-amplitude coupling pre and post antiepileptic drug administration. Where pre is taken to be the baseline.

\subsubsection{Labeling of Responders and Non-Responders}

Responses to AED treatments were determined from posttreatment iEEG recordings by examining the percentage of long duration discharges exhibiting delta-HFO crossfrequency coupling (Table 1) as measured by $I_{C F C}$.

In order to determine the significance of the $I_{C F C}$ values, we chose to use a method known as bootstrapping. In such a method, many time-series signals, referred to as surrogates, are generated from the original data to test the null hypothesis that the observed $I_{C F C}$ resulted by chance. The hypothesis was tested by randomizing the phases across frequency components of the surrogate signals, while keeping all other statistical properties of the signal constant, and obtaining their $I_{C F C}$. If the $I_{C F C}$ obtained on the original signal is found to be significantly larger than the distribution of $I_{C F C}$ values generated from the ensemble of surrogate signals, then one can be confident to reject the hypothesis.

Surrogate time-series signals were generated by employing amplitude adjusted Fourier transform (AAFT) as described by Theiler et al. [42] which generates a vector of random numbers following the Gaussian distribution which are then rank-ordered according to the same rank-order of the original time-series signal. The fast Fourier transform (FFT) was then applied to the IMFs extracted from the iEEG signal $x(\hat{t})$ using EEMD and each phase was multiplied by $e^{j \vartheta}$, where $\vartheta$ is a random number from a uniform distribution between $[0,2 \pi]$ and $\vartheta(f)=-\vartheta(-f)$. This allowed for the inverse FFT to be a vector of only real values. The resulting real-only inverse FFT output yielded the surrogate time-series.

The $I_{C F C}$ computation was performed on surrogate IMFs using Hilbert transform to obtain $A\left(\hat{t}, f_{H}\right)$ and $\varphi\left(\hat{t}, f_{L}\right)$. The significance of the phase-amplitude couplings were determined from $\mathrm{N}=200$ surrogate time-series samples comparing to see if the $I_{C F C}$ obtained from the original undistorted signal exceeded the $95 \%$ percentile of the $I_{C F C}$ values obtained from surrogate cases.

\subsubsection{Testing}

Performance was evaluated for sensitivity and specificity by performing a Receiver Operating Characteristic (ROC) on the predicted probability scores $[43,44]$. The area under the ROC curve provided a score to quantify the performance. Predictions were further evaluated on an individual basis by counting the number of samples predicting successful treatment outcome over the total number of samples, yielding a likelihood score for treatment efficacy. 
A
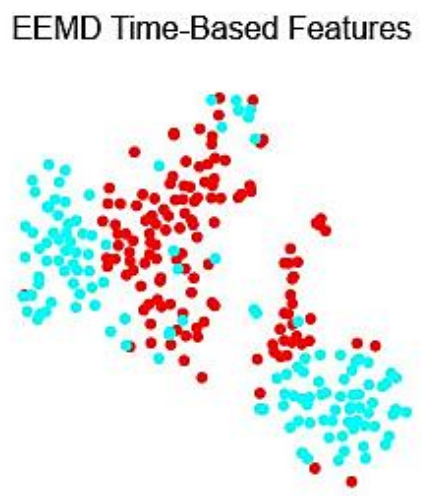

C

\section{Comodulogram Features}

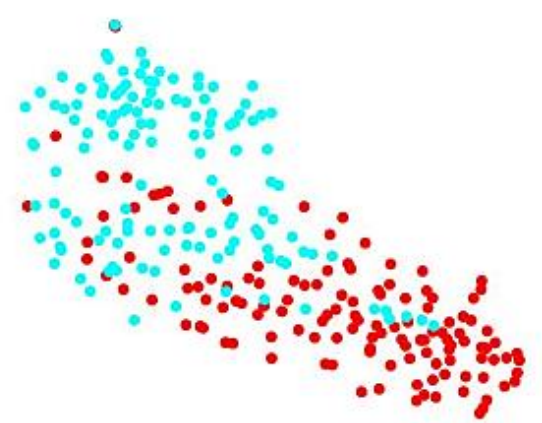

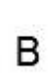

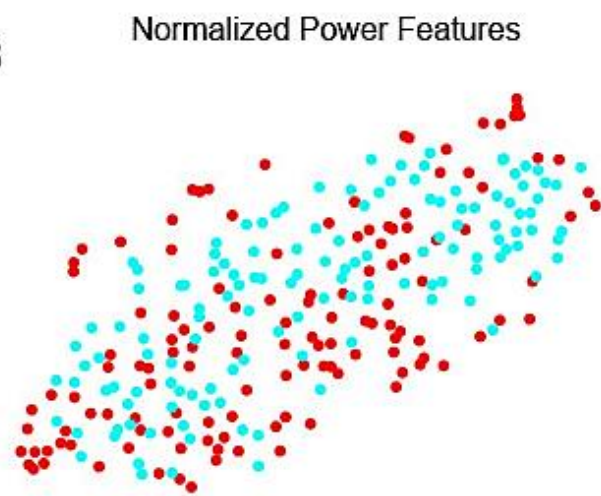

EEMD Time-Based and Comodulogram Features

\begin{abstract}
Fig. 5. Low dimensional feature projections for the antiepileptic drug THIP (one of four tested) shows non-responders in red and responders in cyan. A) EEMD time-based features required a nonlinear separation, B) Normalized power features showed no separability, C) Comodulograms are linearly separable with minor overlapping errors, and D) Feature set representing the concatenation of EEMD time-based and comodulogram features achieves the most linear separation of responders and non-responders.
\end{abstract}

\section{RESULTS}

\subsection{Pathology linked to delta - HFO Coupling}

The genetically modified, Mecp2-deficient mouse model of Rett Syndrome shows presence of epileptiform discharges (Figure 1a). This genetic model provides a means to evaluate iEEG features pre- and post- mеср 2 gene reactivation (prerescue), and provides a means of identifying the link to pathology. EEMD and comodulograms analyses of pre-rescue discharges highlight the presence of delta $(2-5 \mathrm{~Hz})$, theta (6$10 \mathrm{~Hz})$ and fast ripple $(400-600 \mathrm{~Hz})$ rhythms with the fast ripple rhythms modulated by the phase of the delta and theta rhythms (Figure 1b, 1c).

Comodulograms generated on typical post-rescue discharges (Figure 1d) reveal that delta $(2-5 \mathrm{~Hz})$ modulated fast ripple $(400-600 \mathrm{~Hz}) \mathrm{HFO}$ presence is abolished, whereas theta $(6-10 \mathrm{~Hz})$ modulated fast ripple HFO is still present in a diminished form after gene reactivation (Figure 1e, 1f). Reactivation of the теср 2 gene increased the lifespan of Mecp2-deficient mice, thus correcting the effects of the pathology (Figure 2a). Recordings of iEEG in Mecp2deficient mice show a progressive increase in discharge occurrence with age, which is reduced post mecp 2 gene reactivation as shown in Figure $2 \mathrm{~b}$. The percentage of discharges with significant delta-HFO modulation is also shown to increase with age and is reduced in post-rescue mice (Figure 2c).

Previous studies show that these rescued mice perform better on physical and cognitive tests, and have improved life spans that come close to the longer lifespans observed in wild types [30] (figure 2a). These results provide strong evidence that the presence of long duration discharges with delta-fast ripple modulation (delta-HFO) are indicators of pathological seizure activity. This suggests that the extent of delta-HFO modulation could be used to score post treatment outcome [45].

\subsection{AED Treatment outcomes varied across subjects}

Delta and fast ripple rhythms were extracted using EEMD to quantify the extent of delta-HFO phase-amplitude coupling in 6 Mecp2-deficient mice. Prior to AED treatment there was an elevated presence of delta-HFO coupling in the long duration discharges $(60 \%+/-9 \%)$, as shown in Table 1. Post AED treatments it was observed that Midazolam, Ganaxolone, THIP showed on average a decrease in deltaHFO modulation, whereas Phenytoin showed an increase in delta-HFO modulation. 


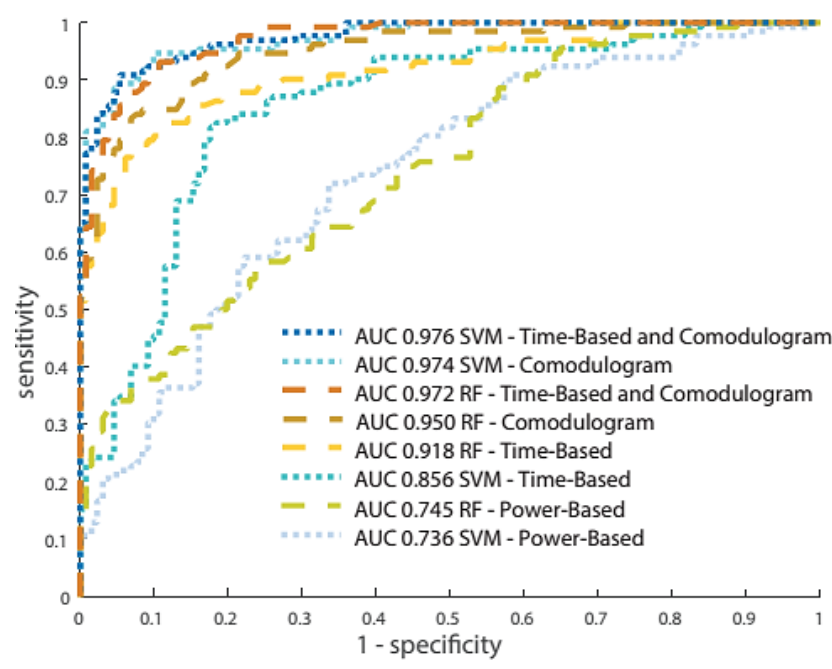

Fig. 6. ROC evaluation of SVM and RF machine learning methodologies across the four feature sets for predicting treatment efficacy for THIP antiepileptic drug. In all cases the normalized power features performed inferior to other features. EEMD time-based achieved reasonable performance and comodulograms features achieved the best results. The combined EEMD time-based and comodulogram feature vector achieved a marginal improvement on the comodulogram results.

Examining the delta-HFO modulation in long duration discharges on a per subject basis revealed high variability in treatment outcome. For example, while half of the subjects were effectively treated with Midazolam, the other half either became worse or showed little to no change. A similar finding was observed for the other three AEDs, highlighting that treatment outcome is highly dependent on the individual subject.

\subsection{Combined features provide greatest class separation}

T-SNE low dimensional projections of EEMD time-based, normalized power and phase-amplitude coupling (comodulogram) features are shown in Figure 4. Samples are colored according to which subjects they came from. The projections revealed that selection of features is vital in achieving class separation and thus has a profound effect on determining treatment outcome.

The normalized power feature projections did not show any clustering by individual animal subjects and were the least useful features in terms of separating responders and nonresponders (Figure 4b). The EEMD time-based and comodulogram features achieved the best separability with distinct clusters for each of the animal subjects. The comodulogram showed less pronounced clustering when compared to the EEMD time-based features, however, certain mouse subjects were better defined.

The best results were achieved when both EEMD timebased and comodulogram features were used. This was further validated when the labels were changed from mouse subjects to responders and non-responders. Figure 5 compares responders and non-responders to THIP and it can be seen that EEMD time-based features would lead to non-linear separation requirements which would pose a greater challenge to applying machine learning techniques for prediction. Overall the EEMD time-based and Comodulogram combination produced the best class separation.

\section{4 iEEG-based features predict treatment efficacy}

To determine whether it is possible to determine treatment outcome from the iEEG, we examined different combinations of features and machine learning algorithms. A sample result of sensitivity versus specificity is shown for the THP training case (Figure 6). The ROCs show similar performance with the SVMs and Random Forests, with the SVMs performing marginally better. The greatest variability in success came from the features selected. In all cases the normalized power features performed poorly, followed next by the time-series features which showed varied success across different drugs. The most consistent performance came from the combined EEMD time-based and comodulogram data sets, reaching accuracy levels of $97.6 \%$.

The results summarizing the combined time-series and comodulogram features trained with SVMs and RFs are shown in Figure 7. The figure shows the percentage of discharges that are correctly identified over all the discharges tested. A higher percentage indicates that the treatment was predicted to be more effective and likely to lead to a favorable outcome. The green and brown bars (responder and nonresponder respectively) indicate the actual outcome for the specific drug. The results for the SVM based predictions (Figure 7a) correlate closely to the treatment outcomes percentages shown in Table 1. Furthermore, in many cases the predicted results matched the uncertainty in treatment efficacy. For example, in Figure 7a, mouse 2 was predicted to have a $67 \%$ likelihood of effective treatment outcome to Midazolam. This matches closely to the expected delta-HFO outcome score of $54 \%$ seen in Table 1. Even though Midazolam was not effective on mouse 2, it was not completely terrible either. The RF based predictions (Figure 7b) also performed well overall, but there are a couple instances where the prediction was completely wrong. For example, the predictions of outcome for the Ganaxolone drug for mouse 2 showed a $100 \%$ success rate, which we know was not successful. Aside from some misclassifications, RF performed similarly to SVM, and in some cases it was slightly better.

Overall, the SVMs were the machine learning method of choice. The predicted likelihood scores obtained with SVMs aligned well with the expected labels and there were no obvious misclassifications.

\section{DisCUSSION}

\section{1 iEEG-based features predict epilepsy treatment outcome}

Patient variability is a serious challenge to selecting treatments for epilepsy. Often antiepileptic drug treatments are cycled through until an effective treatment can be found, and with over two dozen commonly prescribed AEDs available, it can be a cumbersome process. There are certain AEDs that have been found to be statistically more likely to lead to a successful treatment outcome and it is those AEDs that typically are tried first. However, the likelihood of a 


\section{Support Vector Machine Predictions}
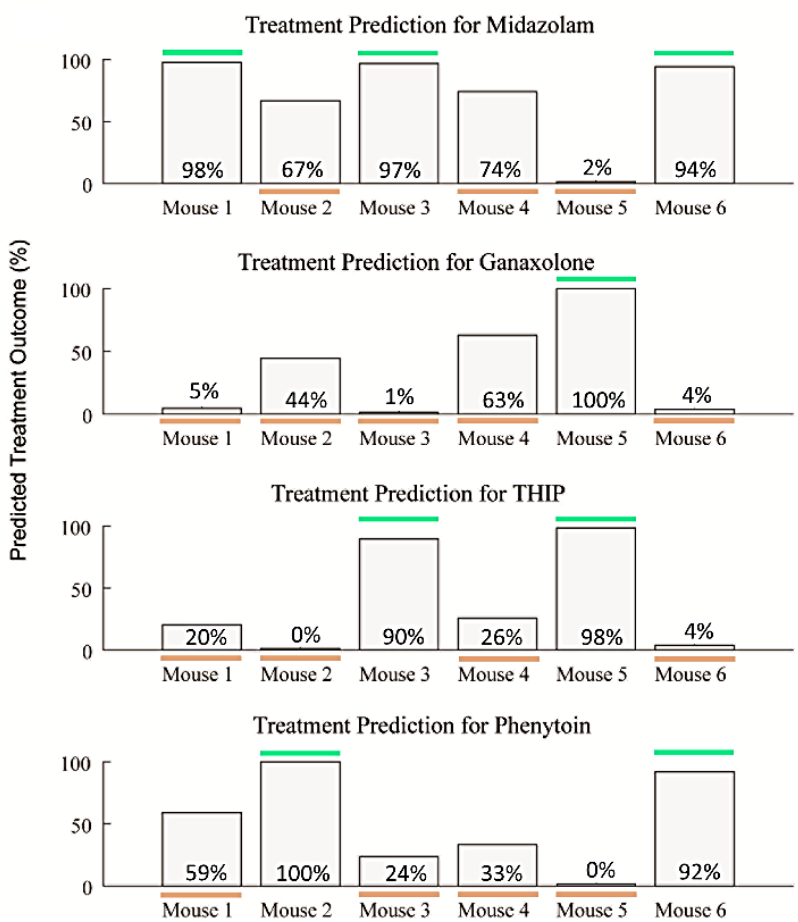

B
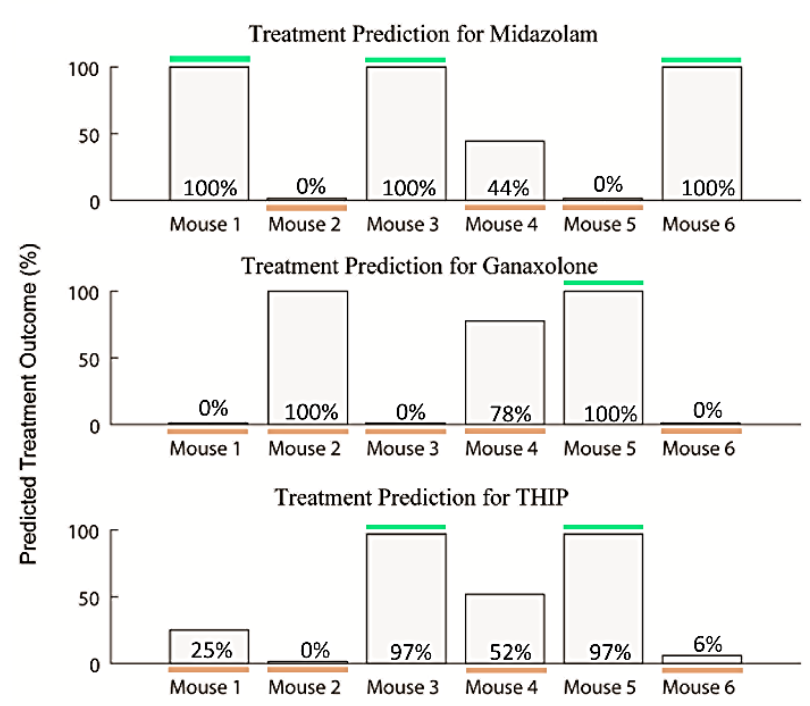

Treatment Prediction for Phenytoin

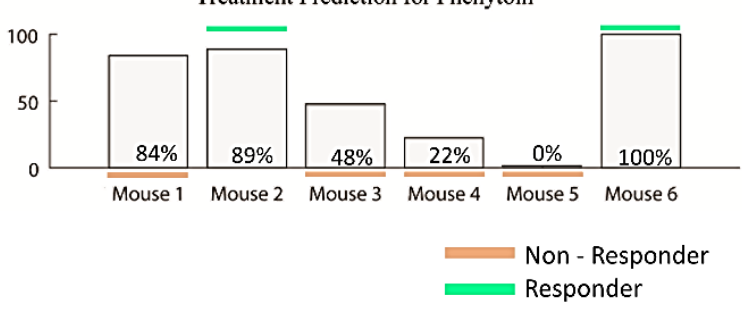

Fig. 7. Predicted likelihood of favorable treatment outcome across four commonly used AEDs using SVM and RF machine learning algorithms. Green bars indicate patient was successfully treated by specific AED, and brown bars indicate unsuccessful treatment. A) SVMs predictions accurately predicted treatment outcome for all AEDs. B) RFs had comparable prediction results, with misclassifications for Ganaxolone treatment for mice 2 and 4 and Phenytoin for moues 1 . successful treatment reduces with each round of AED application [46], possibly due to patient desensitization to AEDs which happens over time.

Our findings reveal that iEEG-based features, obtained from the subject, can be used to predict treatment outcome to anti-epileptic drugs (AEDs). By indicating which patients would be unresponsive to certain AEDs it would avoid unnecessary testing and would have a profound effect on patient quality of life.

Approximately $20-40 \%$ of the patients are unresponsive to AED treatment [2,3], which is a diagnosis that can only be ascertained after an exhaustive search over many AEDs. This is particularly evident in cases of Rett Syndrome where patients generally do not respond favorably to commonly prescribed AEDs $[19,20]$. In these hard to treat cases, it would be beneficial to pre-identify the most likely AED for each subject based on their biomarkers rather than general population statistics.

\subsection{Critical LFO-HFO Ranges for Tracking Efficacy of Therapies}

In recent years there has been extensive research on detection of seizure pathology through the use of biomarkers based on time-frequency power distributions [9], phase phase coupling [10] and phase-amplitude coupling [8]. In particular, Guirgis et al., showed the presence of delta-HFO to be a marker for epileptogenesis [8].

Interactions between theta and gamma frequency ranges have frequently been identified with memory coding [47, 48] and cognitive processing during performance task [34]. These cognitive links suggest that high levels of CFC is not pathological on its own, but rather the specific frequencies of interactions that are linked to the pathology. In the case of memory coding, it has been shown that even under error trials, where presumably there is miscoding of information, there is still a measured elevation of the CFC index [34].

The results presented here further support the importance of the interactions of the rhythms. Specifically the interactions between the delta and HFO frequency ranges [27] are distinct from theta and HFO frequency ranges (Figure 1c, f), for tracking improvements in lifespan longevity (Figure 2).

\subsection{Comodulogram as a critical feature for prediction}

Our findings show that variants of the above reported biomarkers can also be used as features to predict treatment outcome of AEDs. When comparing features across individual mice using t-SNE it was noted that EEMD timebased and comodulogram features produced distinct clusters with minor overlapping across other mouse clusters (Figure 4). This suggests that such features managed to extract some latent information related to subject variability. On the other hand, power based features showed no discernable clusters, which was evident in their poor performance in testing. This could be due to the short duration of the fast ripple activity which could have been averaged out over the time window.

Evaluation of three features using ROCs for both SVMs and RFs (Figure 6) showed that comodulogram features 
(AUC 0.974) outperformed those of EEMD time-based (AUC 0.918 ) and normalized power (AUC 0.745).

\subsection{Comparison of Machine Learning Algorithms}

On average SVMs and RFs performed equally well on the ROC evaluations. However when the techniques were evaluated to predict treatment outcome of four different AEDs, SVMs were found to predict the treatment outcome of outliers found in RF predictions. For example, in Figure 7, RF predictions of treatment outcome for Ganaxolone applied on mouse 2 was close to $100 \%$, when it should have been closer to $0 \%$, whereas SVMs predicted $44 \%$. Similarly, for Phenytoin the prediction for mouse 1 was $84 \%$ when it should have been closer to $0 \%$, whereas SVMs predicted $59 \%$. Generally, SVMs estimated $90 \%$ or greater likelihood scores only for successful treatments.

When the t-SNE projections were examined in terms of responders and non-responders, two classes were well clustered and separable. The EEMD time-based features typically yielded a non-linear separation between responders and non-responders, whereas comodulogram were linearly separable. This may explain why the RFs, which are ideally suited for non-linearly separable classifications, seemed to do better than SVMs on EEG time-based classifications.

\section{CONCLUSION}

The focus of this work was to investigate iEEG-based features, first as biomarkers of epilepsy in a Mecp2-deficient mouse model of Rett Syndrome, and second, as predictors of treatment outcome to AEDs. Specifically, cross-frequency coupling features, in combination with EEMD time-based features, yielded the best likelihood scores of treatment outcome in a machine learning framework. The work presented here may one day lead to more efficient selection of antiepileptic drug treatments from biomarkers obtained from patients, leading to a customized treatment that could lead to more effective treatment outcomes. These techniques are scalable, hence could be extended to treatments focusing on diet, surgery and neuromodulation [49], which could help epileptologists in choosing the most appropriate therapy for their patients.

\section{ACKNOWLEDGMENT}

This work was supported by grants from the Natural Sciences and Engineering Research Council of Canada and from the Canadian Institutes of Health Research.

\section{REFERENCES}

[1] D. Hirtz, D. Thurman, K. Gwinn-Hardy, M. Mohamed, A. Chaudhuri, and R. Zalutsky, "How common are the "common" neurologic disorders?," Neurology, vol. 68, no. 5, pp. 326-337, 2007.

[2] J. Jacobs, M. Zijlmans, R. Zelmann, A. Olivier, J. Hall, J. Gotman, and F. Dubeau, "Value of electrical stimulation and high frequency oscillations $(80-500 \mathrm{~Hz})$ in identifying epileptogenic areas during intracranial EEG recordings," Epilepsia, vol. 51, no. 4, pp. 573-582, 2010.

[3] W. Löscher, and C. Brandt, "High seizure frequency prior to antiepileptic treatment is a predictor of pharmacoresistant epilepsy in a rat model of temporal lobe epilepsy," Epilepsia, vol. 51, no. 1, pp. 8997, 2010.

[4] C. f. D. Control, and Prevention, "Prevalence of epilepsy and healthrelated quality of life and disability among adults with epilepsy--South Carolina, 2003 and 2004," MMWR. Morbidity and mortality weekly report, vol. 54, no. 42, pp. 1080, 2005.

[5] C. f. D. Control, and Prevention, "Health-related quality of life among persons with epilepsy--Texas, 1998," MMWR. Morbidity and mortality weekly report, vol. 50, no. 2, pp. 24, 2001.

[6] E. Ben-Menachem, J. W. Sander, M. Privitera, and F. Gilliam, "Measuring outcomes of treatment with antiepileptic drugs in clinical trials," Epilepsy Behav, vol. 18, no. 1-2, pp. 24-30, May, 2010.

[7] G. Avanzini, "Is tolerance to antiepileptic drugs clinically relevant?," Epilepsia, vol. 47, no. 8, pp. 1285-1287, 2006.

[8] M. Guirgis, Y. Chinvarun, M. Del Campo, P. L. Carlen, and B. L. Bardakjian, "Defining regions of interest using cross-frequency coupling in extratemporal lobe epilepsy patients," J Neural Eng, vol. 12, no. 2, pp. 026011, Apr, 2015.

[9] J. D. Jirsch, E. Urrestarazu, P. LeVan, A. Olivier, F. Dubeau, and J. Gotman, "High-frequency oscillations during human focal seizures," Brain, vol. 129, no. Pt 6, pp. 1593-608, Jun, 2006.

[10] M. Cotic, O. C. Zalay, Y. Chinvarun, M. del Campo, P. L. Carlen, and B. L. Bardakjian, "Mapping the coherence of ictal high frequency oscillations in human extratemporal lobe epilepsy," Epilepsia, vol. 56, no. 3, pp. 393-402, Mar, 2015.

[11] J. Jacobs, P. LeVan, R. Chander, J. Hall, F. Dubeau, and J. Gotman, "Interictal high-frequency oscillations $(80-500 \mathrm{~Hz})$ are an indicator of seizure onset areas independent of spikes in the human epileptic brain," Epilepsia, vol. 49, no. 11, pp. 1893-907, Nov, 2008.

[12] M. Zijlmans, J. Jacobs, R. Zelmann, F. Dubeau, and J. Gotman, "Highfrequency oscillations mirror disease activity in patients with epilepsy," Neurology, vol. 72, no. 11, pp. 979-986, Mar, 2009.

[13] A. Khodayari-Rostamabad, G. M. Hasey, D. J. Maccrimmon, J. P. Reilly, and H. de Bruin, "A pilot study to determine whether machine learning methodologies using pre-treatment electroencephalography can predict the symptomatic response to clozapine therapy," Clin Neurophysiol, vol. 121, no. 12, pp. 1998-2006, Dec, 2010.

[14] A. Khodayari-Rostamabad, J. P. Reilly, G. M. Hasey, H. de Bruin, and D. J. Maccrimmon, "A machine learning approach using EEG data to predict response to SSRI treatment for major depressive disorder," Clin Neurophysiol, vol. 124, no. 10, pp. 1975-85, Oct, 2013.

[15] A. Alkan, E. Koklukaya, and A. Subasi, "Automatic seizure detection in EEG using logistic regression and artificial neural network," $J$ Neurosci Methods, vol. 148, no. 2, pp. 167-76, Oct 30, 2005.

[16] A. Shoeb, H. Edwards, J. Connolly, B. Bourgeois, S. T. Treves, and J. Guttag, "Patient-specific seizure onset detection," Epilepsy Behav, vol. 5, no. 4, pp. 483-98, Aug, 2004.

[17] J. A. Dian, S. Colic, Y. Chinvarun, P. L. Carlen, and B. L. Bardakjian, "Identification of brain regions of interest for epilepsy surgery planning using support vector machines," Conf Proc IEEE Eng Med Biol Soc, vol. 2015, pp. 6590-3, Aug, 2015.

[18] R. E. Amir, I. B. Van den Veyver, M. Wan, C. Q. Tran, U. Francke, and H. Y. Zoghbi, "Rett syndrome is caused by mutations in X-linked MECP2, encoding methyl-CpG-binding protein 2," Nature Genetics, vol. 23, no. 2, pp. 185-188, Oct, 1999.

[19] B. Hagberg, J. Aicardi, K. Dias, and O. Ramos, "A progressive Syndrome of Autism, Dementia, Ataxia, and Loss of Purposeful Hand Use in Girls: Rett's Syndrome: Report of 35 Cases," Ann Neurol, vol. 14, pp. 471-479, 1983.

[20] P. Huppke, K. Kohler, K. Brockmann, G. M. Stettner, and J. Gartner, "Treatment of epilepsy in Rett syndrome," Eur J Paediatr Neurol, vol. 11, no. 1, pp. 10-6, Jan, 2007.

[21] B. P. Jung, D. G. Jugloff, G. Zhang, R. Logan, S. Brown, and J. H. Eubanks, "The expression of methyl $\mathrm{CpG}$ binding factor MeCP2 correlates with cellular differentiation in the developing rat brain and in cultured cells," Journal of neurobiology, vol. 55, no. 1, pp. 86-96, 2003.

[22] M. Chahrour, and H. Y. Zoghbi, "The story of Rett syndrome: from clinic to neurobiology," Neuron, vol. 56, no. 3, pp. 422-437, 2007.

[23] J. Guy, B. Hendrich, M. Holmes, J. E. Martin, and A. Bird, "A mouse Mecp2-null mutation causes neurological symptoms that mimic Rett syndrome," Nature Genetics, vol. 27, no. 3, pp. 322-326, Mar, 2001.

[24] R. G. Wither, S. Colic, C. Wu, B. L. Bardakjian, L. Zhang, and J. H. Eubanks, "Daily rhythmic behaviors and thermoregulatory patterns are 
disrupted in adult female MeCP2-deficient mice," PLoS One, vol. 7, no. 4, pp. e35396, 2012.

[25] J. A. D'Cruz, C. Wu, T. Zahid, Y. El-Hayek, L. Zhang, and J. H. Eubanks, "Alterations of cortical and hippocampal EEG activity in MeCP2-deficient mice," Neurobiol Dis, vol. 38, no. 1, pp. 8-16, Apr, 2010.

[26] S. Colic, M. Lang, R. G. Wither, Z. Liang, J. H. Eubanks, and B. L. Bardakjian, "Characterization of HFOs in short and long duration discharges recorded from in-vivo MeCP2-deficient mice," Engineering in Medicine and Biology Society (EMBC), 2014 36th Annual International Conference of the IEEE, pp. 4603-4606, 2014.

[27] S. Colic, M. Lang, R. G. Wither, Z. Liang, J. H. Eubanks, and B. L. Bardakjian, "Gene Reactivation Diminishes Delta-Modulated High Frequency Oscillations During Seizure-Like Events in Mecp2Deficient Mice," Neural Engineering (NER), 2015 7th International IEEE/EMBS Conference on. IEEE, 2015.

[28] Y. Asaka, D. G. M. Jugloff, L. A. Zhang, J. H. Eubanks, and R. M. Fitzsimonds, "Hippocampal synaptic plasticity is impaired in the Mecp2-null mouse model of Rett syndrome," Neurobiol Dis, vol. 21, no. 1, pp. 217-227, Jan, 2006.

[29] D. G. Jugloff, R. Logan, and J. H. Eubanks, "Breeding and maintenance of an Mecp2-deficient mouse model of Rett syndrome," J Neurosci Methods, vol. 154, no. 1-2, pp. 89-95, Jun 30, 2006.

[30] M. Lang, R. G. Wither, S. Colic, C. Wu, P. P. Monnier, B. L. Bardakjian, L. Zhang, and J. H. Eubanks, "Rescue of behavioral and EEG deficits in male and female Mecp2-deficient mice by delayed Mecp2 gene reactivation," Hum Mol Genet, vol. 23, no. 2, pp. 303-318, 2014.

[31] C. Wu, M. Wais, T. Zahid, Q. Wan, and L. Zhang, "An improved screwfree method for electrode implantation and intracranial electroencephalographic recordings in mice," Behav Res Methods, vol. 41, no. 3, pp. 736-41, Aug, 2009.

[32] S. Colic, R. G. Wither, L. Zhang, J. H. Eubanks, and B. L. Bardakjian, "Characterization of seizure-like events recorded in vivo in a mouse model of Rett syndrome," Neural Networks, vol. 46, pp. 109-115, 2013.

[33] I. Daubechies, "Orthonormal bases of compactly supported wavelets," Communications on pure and applied mathematics, vol. 41, no. 7, pp. 909-996, 1988.

[34] A. B. Tort, R. W. Komorowski, J. R. Manns, N. J. Kopell, and H. Eichenbaum, "Theta-gamma coupling increases during the learning of item-context associations," Proc Natl Acad Sci U S A, vol. 106, no. 49, pp. 20942-7, Dec 8, 2009.

[35] Z. WU, and N. E. HUANG, "ENSEMBLE EMPIRICAL MODE DECOMPOSITION: A NOISE-ASSISTED DATA ANALYSIS METHOD," Advances in Adaptive Data Analysis, vol. 01, no. 01, pp. $1-41,2009$.

[36] B. R. Greene, S. Faul, W. P. Marnane, G. Lightbody, I. Korotchikova, and G. B. Boylan, "A comparison of quantitative EEG features for neonatal seizure detection," Clin Neurophysiol, vol. 119, no. 6, pp. 1248-61, Jun, 2008.

[37] L. Van der Maaten, and G. Hinton, "Visualizing data using t-SNE," Journal of Machine Learning Research, vol. 9, no. 2579-2605, pp. 85, 2008.

[38] C.-C. Chang, and C.-J. Lin, "LIBSVM: a library for support vector machines," ACM Transactions on Intelligent Systems and Technology (TIST), vol. 2, no. 3, pp. 27, 2011.

[39] C. Cortes, and V. Vapnik, "Support-vector networks," Machine Learning, vol. 20, no. 3, pp. 273-297, 1995.

[40] L. Breiman, "Random forests," Machine learning, vol. 45, no. 1, pp. 532,2001

[41] P. Hanchuan, L. Fuhui, and C. Ding, "Feature selection based on mutual information criteria of max-dependency, max-relevance, and minredundancy," Pattern Analysis and Machine Intelligence, IEEE Transactions on, vol. 27, no. 8, pp. 1226-1238, 2005.

[42] J. Theiler, S. Eubank, A. Longtin, B. Galdrikian, and J. D. Farmer, "Testing for nonlinearity in time series: the method of surrogate data," Physica D: Nonlinear Phenomena, vol. 58, no. 1-4, pp. 77-94, 1992.

[43] I. N. Bankman, V. G. Sigillito, R. A. Wise, and P. L. Smith, "Featurebased detection of the K-complex wave in the human electroencephalogram using neural networks," Biomedical
Engineering, IEEE Transactions on, vol. 39, no. 12, pp. 1305-1310, 1992.

[44] K. Woods, and K. W. Bowyer, "Generating ROC curves for artificial neural networks," Medical Imaging, IEEE Transactions on, vol. 16, no. 3, pp. 329-337, 1997.

[45] S. Colic, R. G. Wither, M. Lang, Z. Liang, J. H. Eubanks, and B. L. Bardakjian, "Support vector machines using EEG features of crossfrequency coupling can predict treatment outcome in Mecp2-deficient mice." pp. 5606-5609.

[46] M. Brodie, S. Barry, G. Bamagous, J. Norrie, and P. Kwan, "Patterns of treatment response in newly diagnosed epilepsy," Neurology, vol. 78, no. 20, pp. 1548-1554, 2012.

[47] J. E. Lisman, "Bursts as a unit of neural information: making unreliable synapses reliable," Trends in neurosciences, vol. 20, no. 1, pp. 38-43, 1997.

[48] O. Jensen, and J. E. Lisman, "Novel lists of 7+/-2 known items can be reliably stored in an oscillatory short-term memory network: interaction with long-term memory," Learning \& Memory, vol. 3, no. 2-3, pp. 257263, 1996.

[49] M. T. Salam, J. L. Perez-Velazquez, and R. Genov, "Seizure Suppression Efficacy of Closed-loop Versus Open-loop Deep Brain Stimulation in a Rodent Model of Epilepsy," 2015. 